\title{
Zu Burghardt J, Buess GF: Transanale endoskopische Mikrochirurgie beim Rektumfrühkarzinom. Chir Gastroenterol 2005;21:62-67
}

\author{
Jürgen Weitz Markus W. Büchler \\ Abteilung für Allgemein-, Visceral- und Unfallchirurgie, Chirurgische Universitätsklinik Heidelberg, Deutschland
}

Im multimodalen Therapiekonzept des Rektumkarzinoms nimmt die chirurgische Behandlung einen zentralen Platz ein. Dabei stellt die Vermeidung eines Lokalrezidivs durch adäquate Radikalität unter gleichzeitiger Wahrung von Kontinenz und Lebensqualität eine besondere Herausforderung für den Chirurgen dar [1]. Eine der wesentlichen Weiterentwicklungen der Chirurgie war die Einführung der totalen mesorektalen Exzision (TME) durch Heald; auch ohne adjuvante Strahlentherapie können hierdurch Lokalrezidivraten von weit unter $10 \%$ erreicht werden [2]. Die besondere Relevanz der Vermeidung eines Lokalrezidivs eines Rektumkarzinoms ergibt sich aus der Tatsache, dass eine kurative Resektion eines Lokalrezidivs, wenn überhaupt, nur durch eine ausgedehnte multiviszerale Resektion mit erheblicher Einschränkung der Lebensqualität erreicht werden kann. Die Vermeidung eines Lokalrezidivs ist daher das oberste Gebot; an den extrem niedrigen Lokalrezidivraten die sich mit optimaler Chirurgie erreichen lassen müssen sich daher alle Änderungen der Therapiekonzepte messen. Vor dem Hintergrund der mit der anterioren Rektumresektion auftretenden perioperativen Morbidität sind lokale Therapieverfahren (z.B. transanale endoskopische Mikrochirurgie; TEM) für Rektumfrühkarzinome eingeführt worden. Für Rektumadenome und T1-Karzinome ohne Risikofaktoren stellen lokale Verfahren ein anerkanntes Konzept dar.

Wie so oft in der Entwicklung der Chirurgie wird versucht, weitere Indikationen für eine vorhandene Operationsmethode zu evaluieren. In der Übersichtsarbeit von Burghardt und Buess wird geschlussfolgert:

«Für lokal fortgeschrittene Rektumkarzinome ist eine TEM nach neoadjuvanter Radiochemotherapie möglich. Im Falle eines pT2-Rektumkarzinoms kann eine adjuvante Behandlung das Lokalrezidivrisiko senken.»

Eine derartige Schlussfolgerung, die eine Änderung des anerkannten Therapiekonzepts impliziert, sollte auf einer sorgfäl- tigen Analyse der vorhandenen Evidenz beruhen. Die in der Literatur berichteten Lokalrezidivraten nach lokaler Exzision von T2-Rektumkarzinomen mit adjuvanter Radiatio schwanken, liegen aber deutlich höher als die von Burghardt und Buess zitierten 6\% [3]. Dies ist auch nicht verwunderlich, liegt doch die Inzidenz von Lymphknotenmetastasen bei T2-Rektumkarzinomen bei etwa 12-28\% [3]. In einer Studie aus dem Memorial Sloan Kettering Cancer Center lag beispielsweise die 5- und 10-Jahres-Lokalrezidivrate nach lokaler Exzision von T2-Rektumkarzinomen mit adjuvanter Radiatio bei $24 \%$, eine Senkung der Lokalrezidivrate durch die Bestrahlung war nicht nachzuweisen [4]. Besonders Besorgnis erregend ist, dass nur etwa die Hälfte der Patienten mit einem Rezidiv einer erneuten potentiell kurativen Resektion zugeführt werden konnte und dass die 6-Jahres-Überlebensrate dieser Gruppe bei nur $45 \%$ lag. In einer weiteren Arbeit aus der gleichen Arbeitsgruppe wurden 49 Patienten untersucht die wegen eines Lokalrezidivs nach lokaler Exzision nachoperiert wurden [5]. Bei 55\% dieser Patienten musste eine ausgedehnte multiviszerale Resektion durchgeführt werden. Obwohl bei 47 der 49 Patienten eine R0-Resektion erreicht werden konnte, lag das krankheitsspezifische Überleben bei nur 53\%. Besorgnis erregende Daten sind aktuell auch für T1-Karzinome (low-grade) aus der Cleveland Clinic mit einer Lokalrezidivrate von 17\% publiziert worden [6]. Trotz Versuch der Resektion der Rezidive lag die 5-Jahres-Überlebensrate der Patienten mit Rezidiv bei nur 56,2\%. Diese Daten unterstreichen, dass das Auftreten eines Lokalrezidivs beim Rektumkarzinom immer mit einer äußerst schlechten Prognose verbunden ist, und dies vor dem Hintergrund von initial kleinen, gut resektablen Karzinomen. Die zitierte Studie zu einer Kombination aus neoadjuvanter Radiatio bzw. Radiochemotherapie mit einer lokalen Exzision ist äußerst kritisch zu bewerten, da es sich hierbei um ein selektioniertes Patientengut handelt. Selbstverständlich sprechen einzelne Patienten extrem gut auf

\begin{tabular}{ll}
\hline KARGER & @ 2005 S. Karger GmbH, Freiburg \\
Fax +49 7614520714 & Accessible online at: \\
$\begin{array}{l}\text { E-mail Information@Karger.de } \\
\text { www.karger.com }\end{array}$ & www.karger.com/cga
\end{tabular}

Prof. Dr. Dr. h.c. Markus W. Büchler 
eine neoadjuvante Radiochemotherapie an und können dann möglicherweise auch lokal exzidiert werden (einzelne Autoren führen dann sogar gar keine Operation mehr durch [7]). Hieraus eine allgemeingültige Aussage zu formulieren, wie in der Arbeit von Burghardt und Buess geschehen, erscheint uns jedoch nicht gerechtfertigt. Es muss außerdem darauf hingewiesen werden, dass nicht nur eine Resektion, sondern ebenso eine Radiochemotherapie eine erhebliche Belastung für die Patienten darstellt und exakte Studien bezüglich der Spätfol- gen der Strahlentherapie in der vorgeschlagenen Indikation nicht vorliegen.

Die Behandlung des Rektumkarzinoms muss sich weiterentwickeln. Änderungen der Therapiekonzepte sollten aber nur auf dem Boden von randomisierten Studien erfolgen. Derzeit liegen diese Daten für die Ausweitung einer Indikation für die lokale Therapie von Rektumkarzinomen nicht vor, daher sind die von Burghardt und Buess getroffenen Schlussfolgerungen abzulehnen.

\section{Literatur}

1 Weitz J, Koch M, Debus J, Höhler T, Galle PR, Büchler MW: Colorectal cancer. Lancet 2005;365 153-165.

2 Cecil TD, Sexton R, Moran BJ, Heald RJ: Total mesorectal excision results in low local recurrence rates in lymph node-positive rectal cancer. Dis Colon Rectum 2004; 47:1145-1150.

3 Nastro P, Beral D, Hartley J, Monson J: Local excision of rectal cancer: review of literature. Dig Surg 2005;22:6-15.
4 Paty P, Nash G, Baron P, Zakowski M, Minsky B, Blumberg D, Nathanson D, Guillem J, Enker W, Cohen A, Wong D: Long-term results of local excision for rectal cancer. Ann Surg 2002;236:522-530.

5 Weiser MR, Landmann RG, Wong WD, Shia J, Guillem JG, Temple LK, Minsky BD, Cohen AM, Paty PB: Surgical salvage of recurrent rectal cancer after transanal excision. Dis Colon Rectum 2005; 48:1169-1175.
6 Madbouly K, Remzi F, Erkek B, Senagore A, Baeslach C, Khandwala F, Fazio V, Lavery I: Recurrence after transanal excision of $\mathrm{T} 1$ rectal cancer: should we be concerned? Dis Colon Rectum 2005;48 711-719; discussion 719-721.

7 Habr-Gama A, Perez R, Nadalin W, Sabbaga J, Ribeiro U, Silva e Sousa A, Campos F, Kiss D, Gama-Rodrigues J: Operative versus nonoperative treatment for stage 0 distal rectal cancer following chemoradiation therapy: long-term results. Ann Surg 2004;240:711-717. 